Zbigniew Grande, ul. Chodkiewicza 30, 85-064 Bydgoszcz, Poland

\title{
ON STRONG QUASI-CONTINUITY OF FUNCTIONS OF TWO VARIABLES
}

\author{
Abstract \\ Some properties describing the strong quasicontinuity of functions \\ of one and two variables are considered.
}

\section{Preliminaries}

Let $\mathbb{R}$ be the set of all reals and let $E$ denote $\mathbb{R}$ or $\mathbb{R} \times \mathbb{R}$. For $x \in E$ and for $r>0$ let $K(x, r)=\{t \in X:|t-x|<r\}$. Moreover, let $\mu_{e}(\mu)$ be outer Lebesgue measure (Lebesgue measure) in $E$.

Denote by

$$
\begin{aligned}
& d_{u}(A, x)=\limsup _{h \rightarrow 0} \mu_{e}(A \cap K(x, h)) / \mu(K(x, h)), \\
& \left(d_{l}(A, x)=\liminf _{h \rightarrow 0} \mu_{e}(A \cap K(x, h)) / \mu(K(x, h))\right)
\end{aligned}
$$

the upper (lower) outer density of $A \subset E$ at $x$. A $x \in E$ is called a density point of $A \subset E$ if there exists a measurable (in the sense of Lebesgue) set $B \subset A$ such that $d_{l}(B, x)=1$. The family $\mathcal{T}_{d}=\{A \subset E ; A$ is measurable and every $x \in A$ is a density point of $A\}$ is a topology called the density topology $[2,1,7]$. Moreover, let $\mathcal{T}_{e}$ denote the Euclidean topology in $E$.

\section{Definitions and General Properties}

A function $f: E \rightarrow \mathbb{R}$ has property $A(x)$ at a $x \in E$ (abbreviated $f \in A(x)$ ) if there is an open set $U$ such that $d_{u}(U, x)>0$ and the restriction $f \mid(U \cup\{x\})$ is continuous at $x$. A function $f$ has property $B(x)$ at $x$ (abbreviated $f \in B(x)$ )

Key Words: strong quasicontinuity, strong quasi-equicontinuity, strong cliquishness, density topology, functions of two variables

Mathematical Reviews subject classification: Primary: 26A15, 26B05, 54C08, 54C30

Received by the editors January 23, 1995 
if for every $\eta>0$ we have $d_{u}(\operatorname{int}(\{t:|f(t)-f(x)|<\eta\}), x)>0$, where int $(X)$ denotes the Euclidean interior of $X$.

A function $f$ is strongly quasicontinuous at $x$ (abbreviated $f$ is s.q.c. at $x$ ) (is strongly cliquish at $x$ (abbreviated $f$ is s.c.q. at $x$ )) if for every $\eta>0$ and for every $U \in \mathcal{T}_{d}$ such that $x \in U$ there is a nonempty open set $V$ such that $V \cap U \neq \emptyset$ and $|f(t)-f(x)|<\eta$ for all $t \in U \cap V$ (osc $f<\eta$ on the set $U \cap V)[3]$.

A function $f$ has the Denjoy-Clarkson property (abbreviated $f \in D C P$ ) if it is measurable and for all open sets $I \subset \mathcal{R}, J \subset E$ such that $J \cap f^{-1}(I) \neq \emptyset$ we have $\mu_{e}\left(J \cap f^{-1}(I)\right)>0$.

Moreover, denote by $C(f)$ the set of all continuity points of $f$, by $Q_{s}(f)$ the set of all $x \in E$, at which $f$ is s.q.c., by $A(f)$ the set $\{x \in E ; f \in A(x)\}$ and by $B(f)$ the set $\{x \in E ; f \in B(x)\}$. Obviously, $C(f) \subset A(f) \subset B(f) \subset Q_{s}(f)$.

Example 1 Let $C \subset E$ be a closed, nowhere dense set with $\mu(C)>0$. There is an isolated set $B \subset E \backslash C$ such that the closure $\operatorname{cl}(B) \supset C$. If $f$ is the characteristic function of the set $B$, then $f$ is s.q.c. at every point $x \in E \backslash B$, but $f$ doesn't have property $B(x)$ at any $x \in C$ which is a density point of the set $C$.

Remark 1 There is an everywhere s.q.c. function $f: \mathbb{R} \rightarrow \mathbb{R}$ which is continuous at every $x \neq 0$, and such that $f \notin A(0)$.

Proof. Let $\left\{I_{k, n}: k, n \in N\right\}$ ( $N$ denotes the set of all positive integers) be a family of pairwise disjoint closed intervals such that

- $0 \notin I_{k, n}$ for $k, n \in N$,

- $d_{l}\left(\bigcup_{k \in N} I_{k, n}, 0\right)=2^{-n}$ for $n \in N$.

- if $x_{i} \in I_{k_{i}, n_{i}}$ for $i \in N,\left(k_{i}, n_{i}\right) \neq\left(k_{j}, n_{j}\right)$ for $i \neq j, i, j \in N$, and $\lim _{i \rightarrow \infty} x_{i}=x$, then $x=0$.

Such intervals $I_{k, n}$ exist, since in every interval $(1 /(k+1), 1 / k), k \in N$, we can find disjoint closed intervals $J_{k, i}, i \leq n$, such that $\mu\left(J_{k, i}\right)=2^{-i} / k(k+1)$ for $i \leq n$. Then every sequence $\left(I_{k, n}\right)_{n \in \mathbb{N}}$ of all intervals $J_{k, n}$ and all intervals $-J_{k, n}, n \leq k$ and $k \in \mathbb{N}$, satisfies all required conditions.

Let

$$
f(x)= \begin{cases}1 / n & \text { for } x \in I_{k, n}, k, n \in \mathbb{N} \\ 0 & \text { for } x=0 \\ \text { linear } & \text { otherwise. }\end{cases}
$$


Then $f$ is continuous at every $x \neq 0$. Fix $\eta>0$ and $A \in \mathcal{T}_{d}$ such that $0 \in A$. Let $n \in \mathbb{N}$ be such that $1 / n<\eta$. Then $A \cap \bigcup_{k \in \mathbb{N}} I_{k, n} \neq \emptyset$ and there is $k$ such that $A \cap \operatorname{int}\left(I_{k, n}\right) \neq \emptyset$ and $|f(t)-f(0)|=f(t)=1 / n<\eta$ for $t \in A \cap \operatorname{int}\left(I_{k, n}\right)$. So, $f$ is s.q.c. at 0 .

Assume, to the contrary, that $f \in A(0)$. Then there is an open set $U$ such that $a=d_{u}(U, 0)>0$ and $f \mid(U \cup\{0\})$ is continuous at 0 . Fix $n_{0}$ such that $2^{-n_{0}}<a / 2$. Then $d_{u}\left(\bigcup_{n \leq n_{0} ; k \in \mathbb{N}} I_{k, n}, 0\right) \geq 1-a / 2$ and consequently, for every open set $V$ with $0 \in V$ we have $V \cap U \cap \bigcup_{k \in \mathbb{N} ; n \leq n_{0}} I_{k, n} \neq \emptyset$. Since $f(t) \geq 1 / n_{0}$ for each $t \in \bigcup_{k \in \mathbb{N} ; n \leq n_{0}} I_{k, n}$ and $f(0)=0$, the restricted function $f \mid(U \cup 0)$ is not continuous at 0 . So $f \notin A(0)$.

Remark 2 Observe that for the function $f$ from the proof of Remark 1 we have $A(f) \neq B(f)$, since $f \in B(0)$.

Theorem 1 Let $f \in D C P$. If $f$ is s.q.c. at $x \in E$, then $f \in B(x)$.

Proof. Assume, to the contrary, that $f \notin B(x)$. Then there is a $\eta>0$ such that $d_{u}(\operatorname{int}(\{t:|f(t)-f(x)|<\eta\}), x)=0$. Consequently, $d_{l}(\operatorname{cl}(\{t$ : $|f(t)-f(x)| \geq \eta\}), x)=1$, where $\operatorname{cl}(X)$ denotes the closure of $X$. Let $A \subset$ $\operatorname{cl}(\{t:|f(t)-f(x)| \geq \eta\})$ belong to $\mathcal{T}_{d}$ with $d_{l}(A, x)=1$. There is a countable set $B \subset\{t:|f(t)-f(x)| \geq \eta\}$ such that $A \subset \operatorname{cl}(B)$. Since $f \in D C P$, there is $H \subset\{t:|f(t)-f(x)| \geq \eta / 2\}$ belonging to $\mathcal{T}_{d}$ such that $B \subset \operatorname{cl}(H)$. Then $A \subset \operatorname{cl}(B) \subset \operatorname{cl}(\operatorname{cl}(H))=\operatorname{cl}(H)$ and $F=A \cup H \cup\{x\} \in \mathcal{T}_{d}$. Since $f$ is s.q.c. at $x$, there is an open set $U$ such that $U \cap F \neq \emptyset$ and $|f(t)-f(x)|<\eta / 2$ for every $t \in U \cap F$, contrary to $U \cap H \neq \emptyset$ and $H \subset\{t:|f(t)-f(x)| \geq \eta / 2\}$.

Corollary 1 If $Q_{s}(f)=E$, then $B(f)=E$.

Remark 3 The property DCP is well known in differentiation theory [7] and it can be considered also for nonmeasurable functions. Theorem 1 is true if measurability of $f$ is omitted.

Theorem 2 Let $f: E \rightarrow \mathbb{R}$ and let $A \subset E$ satisfy $\mu(A \backslash B(f))=0$. Then $\mu(A \backslash C(f))=0$.

Proof. Assume, to the contrary, that $\mu_{e}(A \backslash C(f))>0$. Then there is an $\eta>0$ such that $G=\{t \in A \cap B(f)$ : osc $f(t) \geq \eta\}$ is of positive outer measure. By the Lebesgue Density Theorem, $H=\left\{t: d_{u}(G, t)=1\right\}$ is measurable and $H \in \mathcal{T}_{d}$. Fix $x \in H \cap G$. Since $f \in B(x)$, we have

$$
d_{u}(\operatorname{int}(\{t:|f(t)-f(x)|<\eta / 3\}), x)>0 .
$$


So $G \cap \operatorname{int}(\{t:|f(t)-f(x)|<\eta / 3\}) \neq \emptyset$. Let $u \in G \cap \operatorname{int}(\{t:|f(t)-f(x)|<$ $\eta / 3\})$. Since $u \in \operatorname{int}(\{t:|f(t)-f(x)|<\eta / 3\}$, we obtain that osc $f(u) \leq 2 \eta / 3$, contrary to $u \in G$ and osc $f(u) \geq \eta$.

Corollary 2 If $\mu(E \backslash B(f))=0$, then $\mu(E \backslash C(f))=0$.

Corollary 3 If $Q_{s}(f)=E$, then $\mu(E \backslash C(f))=0$.

Remark 4 Observe that $Q_{s}(f) \backslash C(f)$ need not have measure zero (e.g. for the function $f$ from Example 1).

Remark 5 It is obvious that if the functions $f_{n}: E \rightarrow \mathbb{R}, n \in \mathbb{N}$, are s.q.c. at a point $x$ and if the sequence $\left(f_{n}\right)_{n}$ converges uniformly to $f$, then $f$ is also s.q.c. at $x$.

Theorem 3 Let $f: E \rightarrow \mathbb{R}$ be a function such that $Q_{s}(f)=E$. Then there is a sequence of functions $f_{n}, n \in \mathbb{N}$, which converges uniformly to $f$ and for which $A\left(f_{n}\right)=E$ for $n \in \mathbb{N}$.

Proof. We prove that for every $\eta>0$ there is $g: E \rightarrow \mathbb{R}$ such that $A(g)=E$ and $|f(x)-g(x)|<\eta$ for all $x \in \mathbb{R}$. Fix $\eta>0$. By Corollary $3 f$ is almost everywhere continuous. So, $V=\left\{y \in \mathbb{R}: \mu\left(\operatorname{cl}\left(f^{-1}(y)\right)\right)>0\right\}$ is countable. Consequently, the linear space $E_{Q}(V)$ over the field $\mathbb{Q}$ of all rationals generated by $V$ is also countable and there is a $c>0$ which is not in $E_{Q}(V)$. Fix $n \in \mathbb{N}$ with $c<n \eta / 6$. Observe that $\mu\left(\operatorname{cl}\left(f^{-1}((2 k-1) c / n)\right)\right)=0$ for all integers $k$ and $h(x)=(2 k-1) c / n$ if $(2 k-1) c / n \leq f(x)<(2 k+1) c / n$ is almost everywhere continuous and $h(x) \leq f(x)<h(x)+2 c / n<h(x)+\eta / 3$ for every $x \in E$. If $d_{u}\left(\operatorname{int}\left(h^{-1}(h(x))\right), x\right)>0$, set $g(x)=h(x)$. If $d_{u}\left(\operatorname{int}\left(h^{-1}(h(x))\right), x\right)=0$, then set $g(x)=h(x)-2 c / n$.

Evidently, $|f-g| \leq|f-h|+|h-g| \leq 2 c / n+2 c / n<\eta / 3+\eta / 3<\eta$. We will prove that $g \in A(x)$ for every $x \in E$. If $(2 k-1) c / n<f(x)</ 2 k+1) c / n$ for some integer $k$, then there is a $r>0$ such that

$$
(f(x)-r, f(x)+r) \subset((2 k-1) c / n,(2 k+1) c / n)
$$

and, by Corollary $1, d_{u}\left(\operatorname{int}\left(f^{-1}((f(x)-r, f(x)+r))\right), x\right)>0$. Since $g(t)=$ $h(t)=(2 k-1) c / n$ for all $t \in \operatorname{int}\left(f^{-1}((f(x)-r, f(x)+r))\right)$, we obtain that $g \in A(x)$.

Now, let $f(x)=(2 k+1) c / n$ for some integer $k$. If $d_{u}\left(\operatorname{int}\left(h^{-1}(h(x))\right), x\right)>0$ then $g \in A(x)$, because $h$ is almost everywhere continuous. Assume that 
$d_{u}\left(\operatorname{int}\left(h^{-1}(h(x))\right), x\right)=0$. From the definition of $h$, because $h$ is almost everywhere continuous and since $f \in B(x)$, we get

$$
d_{u}\left(\operatorname{int}\left(f^{-1}((f(x)-2 c / n, f(x)))\right), x\right)>0
$$

. Since $g(t)=(2 k-1) c / n$ for all $t \in \operatorname{int}\left(f^{-1}((f(x)-2 c / n, f(x)))\right)$ and for $t=x$, we get $g \in A(x)$.

Now for functions $f, g: E \rightarrow \mathbb{R}$ let $\varrho(f, g)=\min \left(1, \sup _{x \in E}|f(x)-g(x)|\right)$. Moreover, denote by $\mathcal{A}(\mathcal{B})\left(Q_{s}\right)$ the family of all functions $f: E \rightarrow \mathbb{R}$ with $A(f)=E(B(f)=E)\left(Q_{s}(f)=E\right)$.

Observe that $\mathcal{B}=Q_{s}$ is a closed subset of the complete metric space

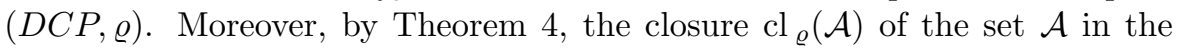
metric $\varrho$ is the same as $\mathcal{B}$.

Remark 6 The set $Q_{s}=\mathcal{B}$ is nowhere dense in the space $(D C P, \varrho)$.

Proof. Since $Q_{s}$ is closed, it suffices to prove that for every $\eta>0$ and for every $f \in Q_{s}$ there is a $g \in D C P \backslash Q_{s}$ such that $\varrho(f, g)<\eta$. Fix $f \in Q_{s}$ and $\eta>0$. Let $F$ be a nowhere dense nonempty set belonging to $\mathcal{T}_{d}$ such that $\operatorname{cl}(F) \subset C(f)$ and let $h$ be the characteristic function of the set $F$. Then $g=f+\eta h / 2 \in D C P \backslash Q_{s}$ and $\varrho(f, g)=\eta / 2<\eta$.

\section{Functions of Two Variables}

Now let $E=\mathbb{R}^{2}$. There are functions $f: E \rightarrow \mathbb{R}$ such that all sections $f_{x}(t)=$ $f(x, t), f^{y}(t)=f(t, y), t, x, y \in \mathbb{R}$, are continuous and $\mu(E \backslash C(f))>0$ [4]. Observe that such functions $f$ are not in $Q_{s}$. However, such functions have the following property $H(x, y)$ at every $(x, y) \in E$.

A function $f: E \rightarrow \mathbb{R}$ has property $H(x, y)(K(x, y))$ at $(x, y)$ if for every $\eta>0$ and for all $U, V \in \mathcal{T}_{d}$ such that $x \in U$ and $y \in V$ there is an open set $W$ such that $W \cap(U \times V) \neq \emptyset$ and $|f(u, v)-f(x, y)|<\eta$ for all $(u, v) \in W \cap(U \times V)$ (osc $f<\eta$ on the set $W \cap(U \times V)$ ).

Theorem 4 If all sections $f_{x}$ and $f^{y}, x, y \in \mathbb{R}$, of $f: E \rightarrow \mathbb{R}$ belong to $Q_{s}$, then $f$ has property $H(x, y)$ at every $(x, y) \in E$.

Proof. Fix $(x, y) \in E$, a real $\eta>0$ and $U, V \in \mathcal{T}_{d}$ such that $x \in U$ and $y \in V$. Since $f^{y} \in B(x)$, there is an open interval $I$ such that $I \cap U \neq \emptyset$ and $|f(t, y)-f(x, y)|<\eta / 4$ for all $t \in I$. Let $F=\operatorname{cl}(I \cap U)$. Since $f_{t} \in B(y)$ for all $t \in F$, for each $t \in F$ there is an open interval $J(t)$ with rational endpoints such that $J(t) \cap V \neq \emptyset$ and $|f(t, v)-f(t, y)|<\eta / 4$ for all $v \in J(t)$. There is 
an open interval $J$ such that $G=\{t \in F: J(t)=J\}$ is of the second category in $F$. Consequently, there is an open interval $I_{1} \subset I$ such that $I_{1} \cap F \neq \emptyset$ and $I_{1} \cap G$ is dense in $I_{1} \cap F$. Evidently, $K=\left(I_{1} \cap U\right) \times(J \cap V) \neq \emptyset$. Fix $(u, v) \in K$ and assume that $|f(u, v)-f(x, y)|>\eta / 2$. Since $f^{v} \in B(u)$, there is an open interval $I_{2} \subset I_{1}$ such that $I_{2} \cap F \neq \emptyset$ and $|f(t, v)-f(x, y)|>\eta / 2$ for all $t \in I_{2}$. Let $s \in I_{2} \cap G$. Then $|f(s, v)-f(x, y)|>\eta / 2$. But

$$
|f(s, v)-f(x, y)| \leq|f(s, v)-f(s, y)|+|f(s, y)-f(x, y)|<\eta / 4+\eta / 4=\eta / 2
$$

This contradiction finishes the proof.

Now, denote by $P_{s}$ the family of all functions $f: E \rightarrow \mathbb{R}$ which are strongly cliquish at every $x \in E$.

Theorem 5 If all sections $f^{y}$ of $f: E \rightarrow \mathbb{R}$ belong to $Q_{s}$ and all sections $f_{x}$ belong to $P_{s}$, then $f$ has property $K(x, y)$ at every $(x, y) \in E$.

Proof. Fix $(x, y) \in E$, and $U, V \in \mathcal{T}_{d}$ such that $x \in U, y \in V$ and $\eta>0$. For every $t \in W=\mathrm{cl}(U)$ there are an open interval $I(t)$ with rational endpoints and a closed interval $J(t)$ with rational endpoints such that $\mu(J(t))<\eta / 2$, $I(t) \cap V \neq \emptyset$ and $f(t, v) \in J(t)$ for every $v \in V \cap J(t)$. Since the family of all pairs of intervals with rational endpoints is countable, there are open intervals $I, L$ and a closed interval $J$ such that $I \cap U \neq \emptyset$ and

$$
A=\{t \in W: I(t)=L, J(t)=J\}
$$

is dense in $I \cap U$. Fix $(u, v) \in(I \times L) \cap(U \times V)$. If $f(u, v) \notin J$, then since $f^{v} \in B(u)$, we obtain that there is a $w \in A \cap U$ such that $f(w, v) \notin J$, contrary to the definition of $A$ and the choice of $I(t)$ and $J(t)$. So, $f(u, v) \in J$ for every $(u, v) \in(I \times L) \cap(U \times V)$ and osc $f \leq \eta / 2<\eta$ on $(I \times L) \cap(U \times V)$.

Problem 1 Suppose that $f: E \rightarrow \mathbb{R}$ has all sections $f^{y} \in Q_{s}$ and all sections $f_{x} \in P_{s}$. Is $f$ in $P_{s}$ ?

Now, denote by $\Phi$ the family of all $f: \mathbb{R} \rightarrow \mathbb{R}$ such that for every nonempty closed set $P$ of positive measure and for every $\eta>0$ there is an open interval $I$ such that $I \cap P \neq \emptyset$ and osc $f<\eta$ on $I \cap P$.

Observe that all Baire 1 functions and all almost everywhere continuous functions are in $\Phi$.

Problem 2 Let $f: E \rightarrow \mathbb{R}$ be such that all sections $f_{x}$ are in $\Phi$ and all sections $f^{y}$ are in $Q_{s}$. Is $f$ in $P_{s}$ ? 
Now we say that the functions $f_{s}: \mathbb{R} \rightarrow \mathbb{R}$, where $s \in S$ and $S$ is a set of indices, are strongly quasi-equicontinuous (abbreviated s.q.ec.) at $x \in \mathbb{R}$ if for every $\eta>0$

$$
d_{u}\left(\operatorname{int}\left(\bigcap_{s \in S}\left(f_{s}\right)^{-1}\left(\left(f_{s}(x)-\eta, f_{s}(x)+\eta\right)\right)\right), x\right)>0 .
$$

Theorem 6 If all sections $f^{y}$ of $f: E \rightarrow \mathbb{R}$ are s.q.c. at every $x$ and if the sections $f_{x}, x \in \mathbb{R}$, are s.q.ec. at every $y$, then $f$ is s.q.c..

Proof. Fix $(x, y) \in E, \eta>0$ and $U \subset E$ belonging to $\mathcal{T}_{d}$ and such that $(x, y) \in U$. Since $f^{y}$ is s.q.c., we get $f^{y} \in B(x)$. Consequently, for the interior $\operatorname{int}\left(\left(f^{y}\right)^{-1}((f(x, y)-\eta / 2, f(x, y)+\eta / 2))\right)=G$ we have $d_{u}(G, x)>0$. Let

$$
H=\operatorname{int}\left(\bigcap_{t \in \mathcal{R}}\left(f_{t}\right)^{-1}((f(t, y)-\eta / 2, f(t, y)+\eta / 2))\right) .
$$

Since the sections $f_{x}$ are s.q.ec. at $y$, we obtain $d_{u}(H, y)>0$. So $G \times H$ is open, $d_{u}((G \times H),(x, y))>0$ and $(G \times H) \cap U \neq \emptyset$. Let $(u, v) \in G \times H$. Then

$$
|f(u, v)-f(x, y)| \leq|f(u, v)-f(u, y)|+|f(u, y)-f(x, y)|<\eta / 2+\eta / 2=\eta
$$

and the proof is complete.

Theorem 7 There is a function $f: E \rightarrow \mathbb{R}$ having continuous sections $f_{x}$ and $f^{y}, x, y \in \mathbb{R}$, such that $\mu(E \backslash C(f))>0$ and for every $\eta>0$, for every $y \in \mathbb{R}$ and for every $U \in \mathcal{T}_{d}$ containing $y$ there is an open interval $I$ such that $I \cap U \neq \emptyset$ and $|f(x, t)-f(x, y)|<\eta$ for all $t \in U \cap I$ and for all $x \in \mathbb{R}$.

Proof. Let $C \subset[0,1]$ be a Cantor set of positive measure. There are pairwise disjoint closed intervals $I_{n} \subset \mathbb{R} \backslash C$ such that

- if $x_{i} \in I_{n_{i}}$ for $i \in \mathbb{N}, I_{n_{i}} \neq I_{n_{j}}$ for $i \neq j$ and $\lim _{i \rightarrow \infty}=x$, then $x \in C$,

- for all $x \in C$ we have $d_{u}\left(\bigcup_{n \in \mathbb{N}} I_{n}, x\right)=0$,

- $C \subset \operatorname{cl}\left(\bigcup_{n \in \mathbb{N}} I_{2 n-1}\right) \cap \operatorname{cl}\left(\bigcup_{n \in \mathbb{N}} I_{2 n}\right)$.

Let $f: E \rightarrow \mathbb{R}$ be a function such that $f(x, y)=0$ if $(x, y) \notin I_{2 n-1} \times I_{2 n}$, $n \in \mathbb{N}, f$ is continuous at every $(x, y) \notin C \times C$ and $f\left(I_{2 n-1} \times I_{2 n}\right)=[0,1]$ for $n \in \mathbb{N}$. Then $f$ satisfies all required conditions. 
Remark 7 Observe that Theorem 7 shows that in Theorem 6 the definition of strong quasi-equicontinuity of sections $f_{x}, x \in \mathbb{R}$, can't be the following: $f_{x}$, $x \in \mathbb{R}$, are s.q.ec. at a point $y$ if for every $\eta>0$ and for every $U \in \mathcal{T}_{d}$ with $y \in U$ there is an open set $V$ such that $V \cap U \neq \emptyset$ and $\left|f_{x}(v)-f_{x}(y)\right|<\eta$ for all $v \in U \cap V$ and $x \in \mathbb{R}$. The function $f$ from Theorem 7 is not in $Q_{s}$, since $\mu(E \backslash C(f))>0$.

Theorem 8 Let $f: E \rightarrow \mathbb{R}$ be a function such that all sections $f_{x}$ are s.q.ec. at every $y$ and all sections $f^{y}$ are almost everywhere continuous. Then $f \in P_{s}$.

Proof. We proceed as in the proof of Theorem 6, but for each $U \in \mathcal{T}_{d}$ we find a $(x, y) \in U$ such that $x$ is a density point of $\{t:(t, y) \in U\}$ and $f^{y}$ is continuous at $x$.

\section{References}

[1] A. M. Bruckner, Differentiation of real functions, Lectures Notes in Math. 659 (1978), Springer-Verlag.

[2] A. M. Bruckner, Differentiation of Integrals, Amer. Math. Monthly, 78 Part II (1971), 1-51.

[3] C. Goffman, C. J. Neugebauer and T. Nishiura, Density topology and approximate continuity, Duke Math. J. 28 (1961), 497-506.

[4] Z. Grande, Measurability, quasicontinuity and cliquishness of functions of two variables, Real Analysis Exchange 20 (1994-95), 744-752.

[5] R. Kershner, The continuity of functions of many variables, Trans. Amer. Math. Soc. 53 (1943), 83-100.

[6] T. Neubrunn, Quasi-continuity, Real Analysis Exch. 14 (1988-89), 259306.

[7] S. Saks, Theory of the integral, Warsaw, 1937. 